\title{
Ground-based photon path measurements from solar absorption spectra of the $\mathrm{O}_{2}$ A-band
}

\author{
Z. Yang ${ }^{a, *}$, P.O. Wennberg ${ }^{a}$, R.P. Cageao ${ }^{\text {b }}$, T.J. Pongetti ${ }^{b}$, G.C. Toon ${ }^{b}$, \\ S.P. Sander ${ }^{\mathrm{b}}$

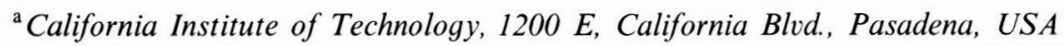 \\ ${ }^{\mathrm{b}}$ Jet Propulsion Laboratory, California Institute of Technology, Pasadena, CA, USA
}

Received 6 October 2003; accepted 13 March 2004

\begin{abstract}
High-resolution solar absorption spectra obtained from Table Mountain Facility (TMF, $34.38^{\circ} \mathrm{N}, 117.68^{\circ} \mathrm{W}$, $2286 \mathrm{~m}$ elevation) have been analyzed in the region of the $\mathrm{O}_{2}$ A-band. The photon paths of direct sunlight in clear sky cases are retrieved from the $\mathrm{O}_{2}$ absorption lines and compared with ray-tracing calculations based on the solar zenith angle and surface pressure. At a given zenith angle, the ratios of retrieved to geometrically derived photon paths are highly precise $(\sim 0.2 \%)$, but they vary as the zenith angle changes. This is because current models of the spectral lineshape in this band do not properly account for the significant absorption that exists far from the centers of saturated lines. For example, use of a Voigt function with Lorentzian far wings results in an error in the retrieved photon path of as much as $5 \%$, highly correlated with solar zenith angle. Adopting a super-Lorentz function reduces, but does not completely eliminate this problem. New lab measurements of the lineshape are required to make further progress.
\end{abstract}

(C) 2004 Elsevier Ltd. All rights reserved.

Keywords: $\mathrm{O}_{2}$ A-band; Photon path; Solar absorption spectra

\section{Introduction}

The A-band is the strongest absorption band of $\mathrm{O}_{2}$ in the visible and IR region. Its importance for atmospheric remote sensing lies not in measuring $\mathrm{O}_{2}$ itself (the mixing ratio is well known), but rather in its use of determining the photon paths traversed through the atmosphere. Because the A-band has the virtue of being in a window region (where the atmosphere is transparent down to the surface) and free from interference of other atmospheric gases (e.g. water vapor), transmission

\footnotetext{
* Corresponding author. Tel.: +1-6263956293; fax: +1-1-6265851917.

E-mail address: yangzh@gps.caltech.edu (Z. Yang).
} 
and reflectance spectra of this band are sensitive to tropospheric ray paths and thus directly relevant to the study of clouds and aerosols from space [1-6]. The A-band spectra have also been used for estimating surface pressure from aircraft [7] and deriving tangent heights for solar occultation observations [8].

Photon path information is extremely important for achieving the required precision of future global-coverage spaceborne measurements of $\mathrm{CO}_{2}$, which are necessary for resolving the global carbon budget. Similar applications on other well-mixed gases (e.g. $\left.\mathrm{CH}_{4}\right)$ are possible. These measurements must have very high precision (e.g. 1 ppmv or $0.3 \%$ for $\mathrm{CO}_{2}$ ) to detect small geographic and temporal changes. The usefulness of the $\mathrm{O}_{2} \mathrm{~A}$-band in improving spaceborne $\mathrm{CO}_{2}$ measurements, however, is predicated on accurate simulation of its absorption spectrum [9].

Retrieval of photon path lengths with sub-percentage precision from atmospheric spectra is always a challenge. For the A-band, the high optical depths mean that most of the lines are saturated from the centers to $\sim 10$ Lorentzian widths, even at the smallest airmass (the ratio of the slant column to the vertical column). The information on the photon paths, therefore, comes largely from the far-wings of these $\mathrm{O}_{2}$ absorption lines where existing laboratory constraints are inadequate. Retrievals from highly saturated spectra also require precise knowledge of both the continuum and zero levels. In addition, temperature information is needed. Finally, the absorption lines in this band are strongly overlapped (especially true for lines in the $\mathrm{R}$ branch), and line-mixing effects could be important.

In this paper, we evaluate the accuracy of photon path retrievals from the A-band spectra in a situation where the photon paths are very well known: ground-based narrow field-of-view observation of direct sunlight in a near-IR spectral region. In this observation geometry, virtually all the measured photons have passed through the atmosphere without being scattered. The photon paths can, therefore, be accurately determined by simple geometric ray tracing. Our main purpose is to develop an algorithm and technique that will allow retrieval of photon path information from the ground-based A-band spectra, and to explore the accuracy-limiting factors.

\section{Measurements}

The data analyzed in this work were obtained with the Fourier Transform Ultraviolet Spectrometer (FTUVS), installed at JPL's Table Mountain Facility, about 40 miles Northeast of Los Angeles [10]. The instrument is designed primarily for use in the visible and near-UV, but is capable of to working in the near-IR with the addition of a Silicon photodiode detector. Measurements were taken on 12 days between March 24 and April 11, 2003, each consisting of 10 scans (5 forward and 5 reverse) obtained in $5 \mathrm{~min}$. The forward and reverse scans were summed separately in order to evaluate any systematic error from different scan directions. Altogether 540 spectra were obtained from 10450 to $17987 \mathrm{~cm}^{-1}$, covering the entire A-band (centered at $13120 \mathrm{~cm}^{-1}$ ), with a resolution of $0.054 \mathrm{~cm}^{-1}$. To minimize the measurement variation, the instrument settings and alignment were adjusted minimally during the 19 day period.

We recorded the beginning and ending times of each measurement with an error smaller than $5 \mathrm{~s}$. This translates to photon path accuracies better than $0.06 \%$ at two airmasses and $0.17 \%$ at five airmasses. The surface pressures were recorded at the same time. We also noted cloudiness and only use spectra taken in clear sky cases. 


\section{Data analysis}

Spectra are analyzed using the GFIT algorithm developed at JPL. This algorithm is able to simultaneously retrieve the column amounts of several molecules from a prescribed spectral window. In the forward model, we represent the atmosphere by 70 levels from 0 to $69 \mathrm{~km}$. Pressure- and temperature-dependent absorption coefficients are computed line-by-line for each level. The atmospheric transmittance $T$ at frequency $v_{i}$ is represented as

$$
T\left(v_{i}\right)=\exp \left[-\sum_{l} p_{l} n_{l} \sum_{g} \lambda_{g} m_{l, g} \sum_{k} s_{l, g, k} f_{l, g, k}\left(v_{i}-v_{l, k}\right)\right],
$$

where $p_{l}$ and $n_{l}$ is the effective optical path length and the total atmospheric number density $\left(\mathrm{mol} \mathrm{cm} \mathrm{c}^{-3}\right.$ ) at the $l$ th level; $m_{l, g}$ is the a priori volume-mixing ratio (VMR) of the $g$ th gas at the $l$ th level; $s_{l, g, k}$ and $f_{l, g, k}$ are the absorption strength $\left(\mathrm{cm}^{2} \mathrm{~mol}^{-1} \mathrm{~cm}^{-1}\right)$ and normalized lineshape function of the $k$ th absorption line of the $g$ th gas at the $l$ th level; and $v_{l, k}$ is the center frequency $\left(\mathrm{cm}^{-1}\right)$ of the $k$ th absorption line at the $l$ th atmospheric level. $\lambda_{g}$ is the scale factor applied to the VMR of the gth gas (for a gas with accurately known mixing-ratio such as $O_{2}, \lambda_{g}$ is effectively the photon path scale factor). The pressure and temperature profiles for each day's spectra are rescaled from NCEP/NCAR reanalysis data, which include temperatures on 17 levels from 1000 to $10 \mathrm{mbar}$ with $2.5^{\circ} \times 2.5^{\circ}$ geographic resolution. Climatological temperature profiles are used for levels with pressure $\leqslant 10$ mbar. The optical path length between each level is calculated based on time-derived astronomical solar zenith angle (SZA), pressure altitude (instead of geometrical altitude to reconcile with the $P-T$ profile), and atmospheric refractive index. All these factors, together with the absorption strengths and lineshape functions, are pre-determined and kept the same during the fitting process.

During the retrieval, the algorithm performs least squares analysis over the spectral window to derive the factors that scale the slant column abundances of the target gases. The fitting residual is defined as

$$
\chi^{2}=\sum_{i=1}^{N_{M}} \frac{\left(Y_{i}^{M}-Y_{i}^{\mathrm{C}}\right)^{2}}{\sigma_{i}^{2}}
$$

$Y_{i}^{M}$ is one measurement in the spectrum and $\sigma_{i}$ is its uncertainty. $Y_{i}^{\mathrm{C}}$ is the corresponding forward model calculation, which is determined by above-atmosphere solar spectrum $Y_{i}^{\mathrm{S}}$, spectral window continuum (approximated linearly as $\left[\alpha+\beta\left(v_{i}-v_{\mathrm{c}}\right)\right]$, with $\alpha$ and $\beta$ the continuum level and tilt, and $v_{\mathrm{c}}$ the window center frequency), zero level offset $\left(Z_{\mathrm{off}}\right)$, instrument lineshape (ILS), frequency shift $(\delta)$ and atmospheric transmittance

$$
Y_{i}^{\mathrm{C}}=Z_{\mathrm{off}}+Y_{i}^{\mathrm{S}}\left[\alpha+\beta\left(v_{i}-v_{\mathrm{c}}\right)\right]\left[I L S\left(v_{i}, \delta\right) \otimes T\left(v_{i}\right)\right] .
$$

$\otimes$ denotes the convolution operation. During retrieval process, both $Y_{i}^{\mathrm{C}}$ and its derivatives with respect to the variables are computed iteratively to find the combination of unknowns $X=\left[\lambda_{1}, \lambda_{2} \ldots\right.$ $\left.\lambda_{G}, \alpha, \beta, \delta, Z_{\text {off }}\right]$ that minimize the residual $\chi^{2}$. 


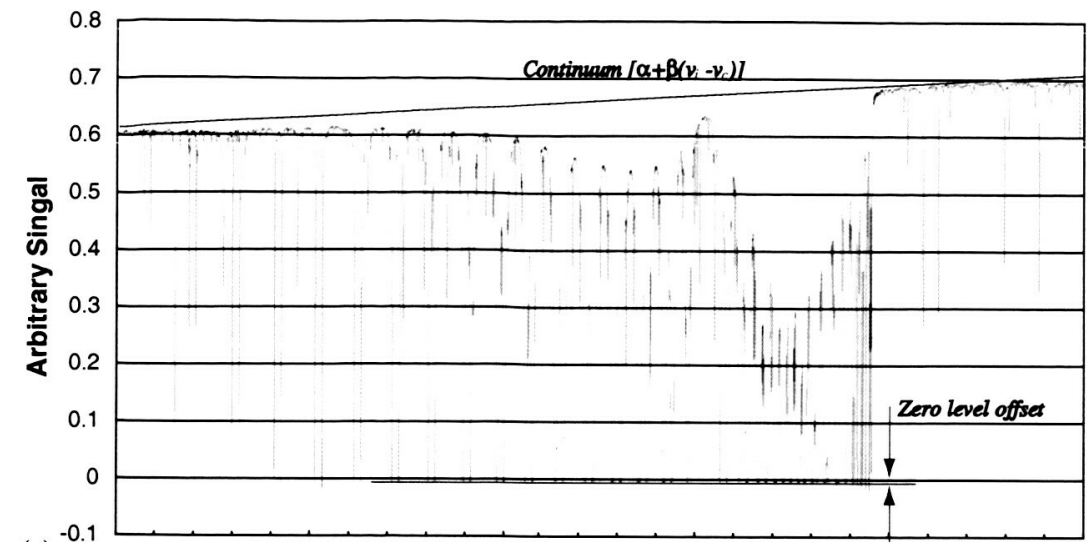

(a)

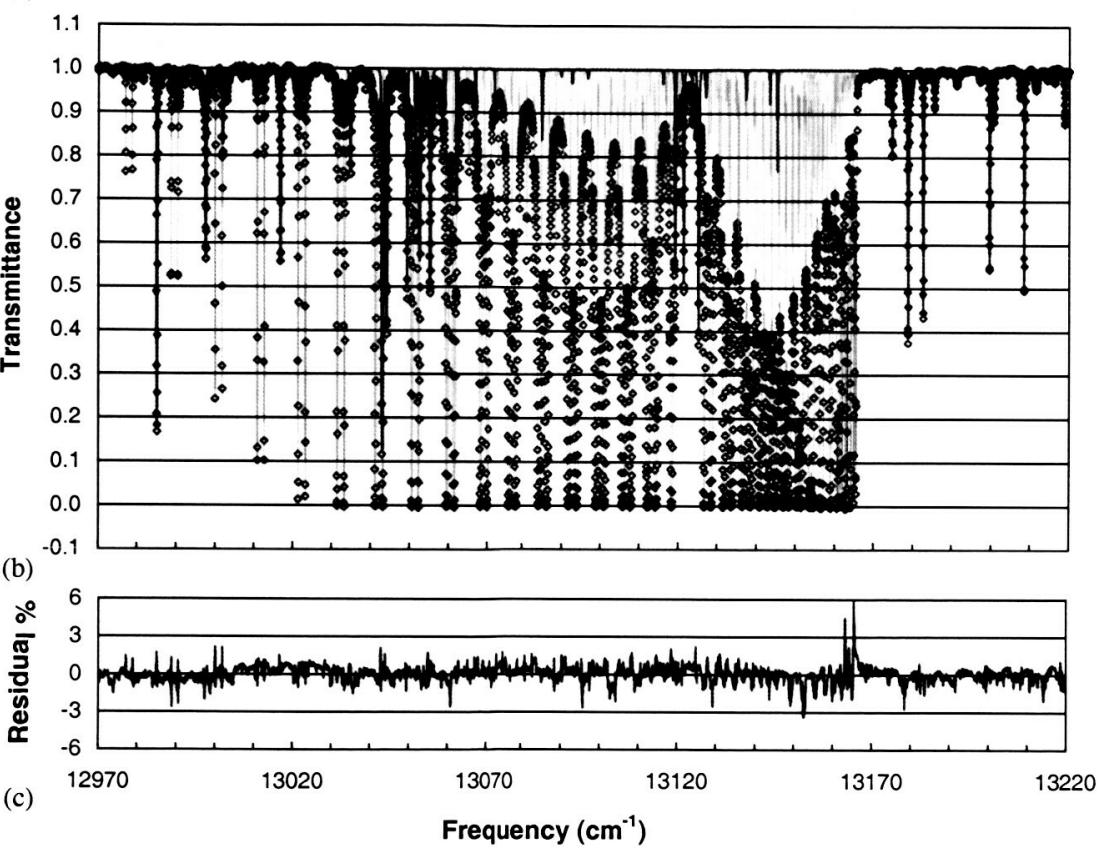

Fig. 1. (a) A TMF $\mathrm{O}_{2}$ A-band (12970-13220 $\mathrm{cm}^{-1}$ ) spectrum measured at $51.5^{\circ} \mathrm{SZA}$ on March 24,2003 . The fitting parameters continuum and zero-level offset are also shown. (b) The result of fitting the same spectrum in the same region. Diamonds are the measurements and black lines are calculated total transmittance. The contributions from $\mathrm{O}^{16} \mathrm{O}^{16}$, $\mathrm{O}^{16} \mathrm{O}^{17}+\mathrm{O}^{16} \mathrm{O}^{18}$, and the solar features are marked by red, cyan, and blue, respectively. Compared with (a), the continuum has been normalized and the zero-level offset has been subtracted. (c) the spectral fitting residual (measured minus calculated). The large peak at $\sim 13165 \mathrm{~cm}^{-1}$ is probably caused by neglecting line-mixing.

Fig. 1(a) shows a raw spectrum and the parameters applied to fit it. The upper line represents the linearized continuum level $\left[\alpha+\beta\left(v_{i}-v_{\mathrm{c}}\right)\right]$ and the lower line represents the $Z_{\mathrm{off}}$. During fitting, the continuum level is determined from regions where the atmospheric absorption is minimal (i.e. beyond the edges of the band), and the $Z_{\text {off }}$ is determined from blacked-out regions, where the 
atmospheric transmittance is known to be zero. The fitted results and residuals of the same spectrum are shown in Figs. 1(b) and (c). The curvature of the continuum is less than $0.2 \%$ judged from residuals, which is small compared with the uncertainty in the gaseous absorption.

Since atmospheric spectra have many saturated lines inside the $\mathrm{O}_{2}$ A-band, even at the lowest airmass, zero level offsets affect the retrieval heavily. An attempt of determining $Z_{\text {off }}$ from the whole A-band gives undue weight to spectral points that are nearly but not exactly blacked out. We thus retrieve zero levels separately from the contrast of non-absorbing and saturated regions inside the A-band, and input the results back into the general retrieval process to determine photon paths. The two-step strategy has reduced the scatter of retrievals from high airmass spectra, but slightly increased the scatter of the low airmass results. Study shows that, because of their narrower saturated regions and consequently larger sensitivity to other factors (e.g. ILS), it is difficult to determine $Z_{\text {off }}$ for low airmass $(<2)$ spectra. The retrieved $Z_{\text {off }}$ values from these spectra scatter much more than the high airmass ones, and induce a spurious correlation between the retrieved scale factors and the $Z_{\text {off }}$ themselves (not shown) after input into the second-step retrieval. Nonetheless, a simple subtraction of this correlation reduces the scatter significantly, and is thus applied to retrievals from all low airmass spectra. The corrections are mostly around $0.1 \%$, although a few can be as large as $0.3 \%$.

A sensitivity test shows that the retrieved photon path scale factor from the A-band has temperature dependence that decreases almost linearly with airmass (from $0.16 \% / \mathrm{K}$ at one airmass to $0.04 \% / \mathrm{K}$ at seven airmasses). The positive temperature dependence indicates that the linewidth rather than the linestrength dominates the overall temperature sensitivity for this heavily saturated band. Since we only use one temperature profile for each day, the diurnal temperature variation at near ground levels could introduce significant error. For this reason, we retrieve the temperature at levels lower than $5 \mathrm{~km}$ using the contrast between the $\mathrm{O}_{2}$ lines of different ground state energies as a correction. Tests using simulated spectra indicate that this strategy accurately retrieves $\mathrm{O}_{2}$ at all airmasses, even when the a priori surface temperature is off by $10 \mathrm{~K}$. This sensitivity test also demonstrated that the retrieved $\mathrm{O}_{2}$ amount is not sensitive to the altitude-dependence of temperature adjust, as either a uniform offset or a top-fixed linear slope. We thereby use a simple offset $T_{\text {off }}$ to change the temperature profiles at altitudes below $5 \mathrm{~km}$.

The positions, intensities, and ground state energies of the $\mathrm{O}^{16} \mathrm{O}^{16}$ A-band spectral absorption lines are from the HITRAN2000 compilation [11,12]. The self- and pressure-broadening coefficients, however, were recalculated from a fit to several lab measurements [12-15] (shown in Fig. 2). In HITRAN2000, the widths of high rotational quantum number (denoted as $J$ hereby) absorption lines were extrapolated from earlier measurements of the lower- $J$ lines. The new linewidth estimates (the orange lines in Fig. 2) substantially reduce the fit residuals especially for the high- $J$ lines. Spectral parameters of $\mathrm{O}^{16} \mathrm{O}^{18}$ and $\mathrm{O}^{16} \mathrm{O}^{17}$ have also been updated [Camy-Peyret, private communication]. To avoid artifacts resulted from inconsistencies among the spectroscopic parameters of the various $\mathrm{O}_{2}$ isotopomers, we retrieve separate scale factors for $\mathrm{O}^{16} \mathrm{O}^{16}$ and $\mathrm{O}^{16} \mathrm{O}^{18}+\mathrm{O}^{16} \mathrm{O}^{17}$, and use only the results from the $\mathrm{O}^{16} \mathrm{O}^{16}$ to determine photon paths.

Line mixing is only prominent for the high- $J$ lines of the R branch band-head $\left(v \sim 13165 \mathrm{~cm}^{-1}\right.$, shown in Fig. 1), although Linda Brown [private communication] has speculated that it likely extends throughout the entire A-band. Since we do not have a good technique to account for this effect, Rbranch $\left(13120-13170 \mathrm{~cm}^{-1}\right)$ is not used. However, the non-absorbing regions on both sides of the A-band are used because they are necessary to derive the spectral continuum. 

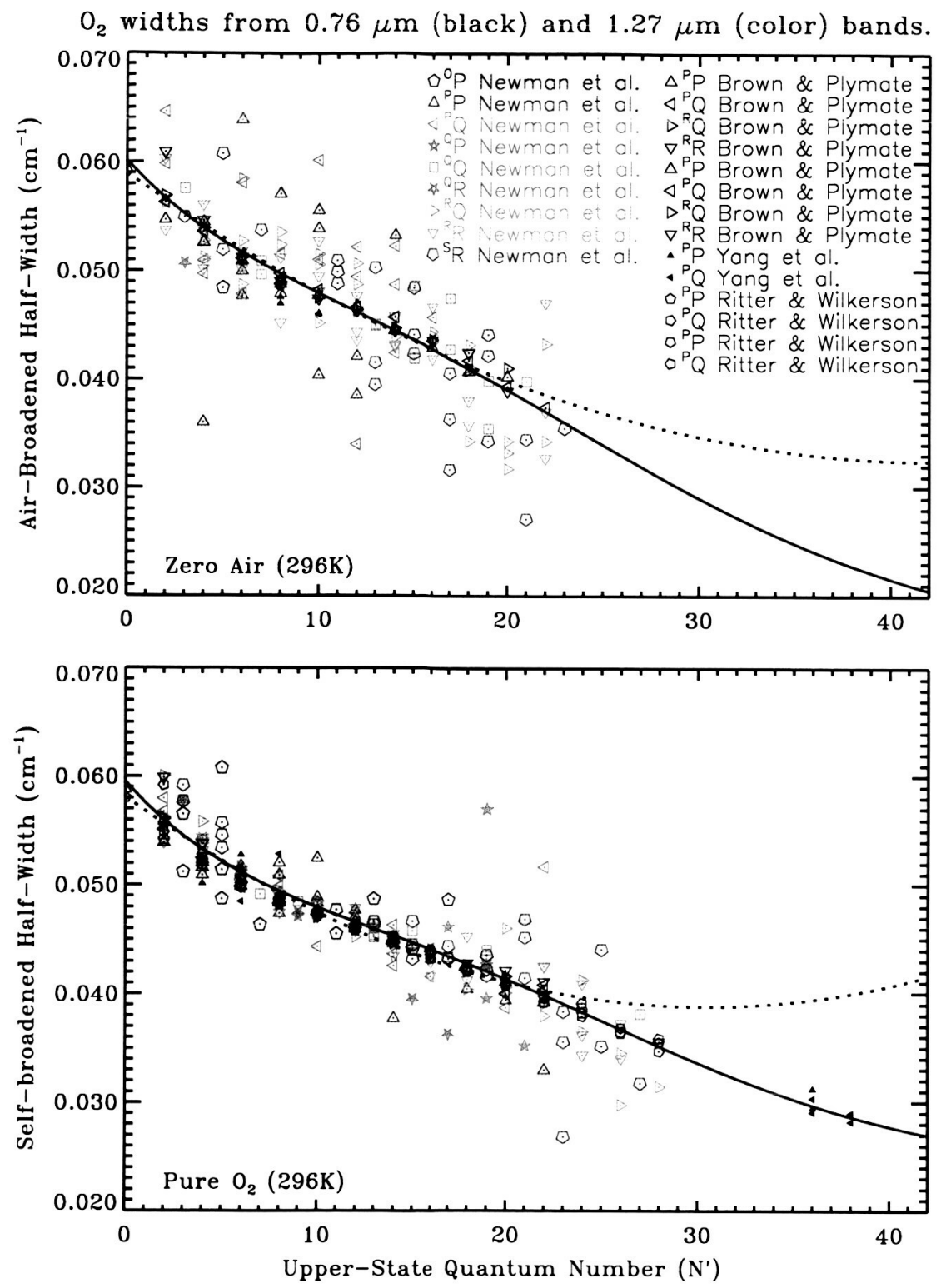

Fig. 2. Laboratory measurements of air- and self-broadened half-width ( $A B H W$ and $S B H W$ ) of $\mathrm{O}_{2}$ A-band (black) and $\mathrm{O}_{2} 1.27 \mu \mathrm{m}$ band (color) absorption lines from different references [13-16]. The fit of the SBHW (orange line in panel (b)) to upper rotational quantum number $\left(N^{\prime}\right)$ is $S B H W=0.02204+\frac{0.03749}{1+0.05428 N^{\prime}-1.19 \times 10^{-3} N^{\prime 2}+2.073 \times 10^{-6} N^{\prime 4}}$. $A B H W$ is then derived from $S B H W$ (orange line in panel (a)) $A B H W=1.012 S B H W / \sqrt{1+\left[\left(N^{\prime}-5\right) / 55\right]^{2}}$. Both diverge from the HITRAN2000 extrapolations (black dashed line) for $N^{\prime}>20$. 


\section{Results and discussion}

Retrieved photon path scale factors (ratios of the retrieved to geometrically derived ray paths) using Voigt function from $\mathrm{O}^{16} \mathrm{O}^{16}$ lines of the A-band FTUVS spectra are plotted versus the airmasses at which they are taken in Fig. 3(a). The results are symmetrical about noon demonstrating no existence of time offsets while recording these spectra. There is also no significant $(<0.1 \%$ in total $)$ difference between the retrievals from the forward and reverse scannings (not shown). At a given airmass, the precision $(1 \sigma \sim 0.2 \%)$ is satisfying, but the spurious correlation between the scale factors and the airmasses is obvious. An examination of the spectral residuals (e.g. Figs. 3(b) and (c)) reveals that the Voigt function gives too low a ratio of far wings to the near wings absorption. Changing linewidths in this formulation neither improves the residuals nor removes the airmass dependence. Considering the Dicke narrowing effect by adopting a hard collision model used to fit A-band lab spectra [12] gives indistinguishable results (not shown) from those of the Voigt lineshape, because Dicke narrowing affects only the line centers, which are blacked out for most of the $\mathrm{O}_{2}$ lines considered here.

In an attempt to improve our retrievals, the super-Lorentz function described by Hirono and Nakazawa [16] has been tested. This function is represented as

$$
f(v)=\frac{A(\eta)}{\pi} \frac{\gamma_{\mathrm{L}}^{\eta-1}}{\left\{\left(v-v_{0}\right)^{2}+\gamma_{\mathrm{L}}^{2}\right\}^{\eta / 2}},
$$

where $\gamma_{\mathrm{L}}$ is the Lorentzian linewidth, $v_{0}$ is the absorption line center frequency, and $1<\eta \leqslant 2$. $A(\eta)$ is the normalization factor. When $\eta=2, f(v)$ is Lorentzian; when $\eta<2, f(v)$ becomes super-Lorentzian, i.e. weaker near the line center and stronger in the far wings (shown in Fig. 4(a)). Although this lineshape is expected to better represent the dominant saturated lines in atmospheric A-band spectra, it provides a poorer representation of the line centers than the Voigt function because it neglects the Doppler effect. In this study, therefore, we apply the super-Lorentz function only to strong low- $J(\leqslant 25)$ lines, which are always saturated at the centers for our spectra, and use Voigt function for other weak lines. Although the division is arbitrary, it gives smaller residuals than the attempt of combining two lineshapes (using Voigt for high altitudes and super-Lorentz for low altitudes).

The comparison of photon path scale factors retrieved using different choices of $\eta$ are shown in Fig. 4(b). As we use smaller $\eta$ value (i.e. increase the far wing absorbtion), both the absolute value of retrieved scale factors and their airmass dependence decreases. In spite of this, there is no single $\eta$-value that completely removes the airmass dependence. For example, at $\eta=1.977$ (which gives the smallest rms for super-Lorentz function fits), the low airmass $(<1.8)$ retrievals are almost airmass-independent, but the other retrievals still increase with airmass; and when $\eta=1.958$, the fitting $\mathrm{rms}$ is larger (but still smaller than those of Voigt function), the airmass-independent region moves to modest airmasses (2.5-4), while low and high airmass retrievals show negative and positive airmass dependences, respectively. An investigation of the fitting residual of saturated lines (not shown) reveals that, compared with the Voigt lineshape, the super-Lorentz lineshape represents the far wings better, but still has the same problem of overestimating the near wings (from line center to $\sim 1.5 \mathrm{~cm}^{-1}$ away), which might be a result of neglecting line-mixing. Besides, this overestimation gets worse for high airmass spectra, which should be responsible for the complex airmass dependences of retrievals. Considering the $2 \%$ accuracy of line intensities [12,14], $\eta=1.977$ gives the most reasonable absolute 


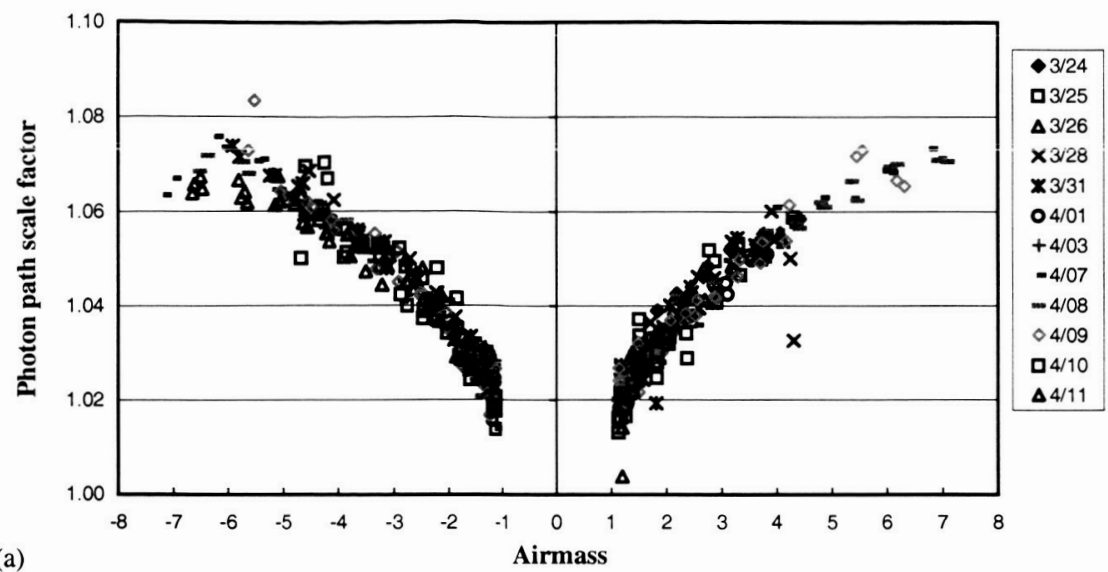

(a)

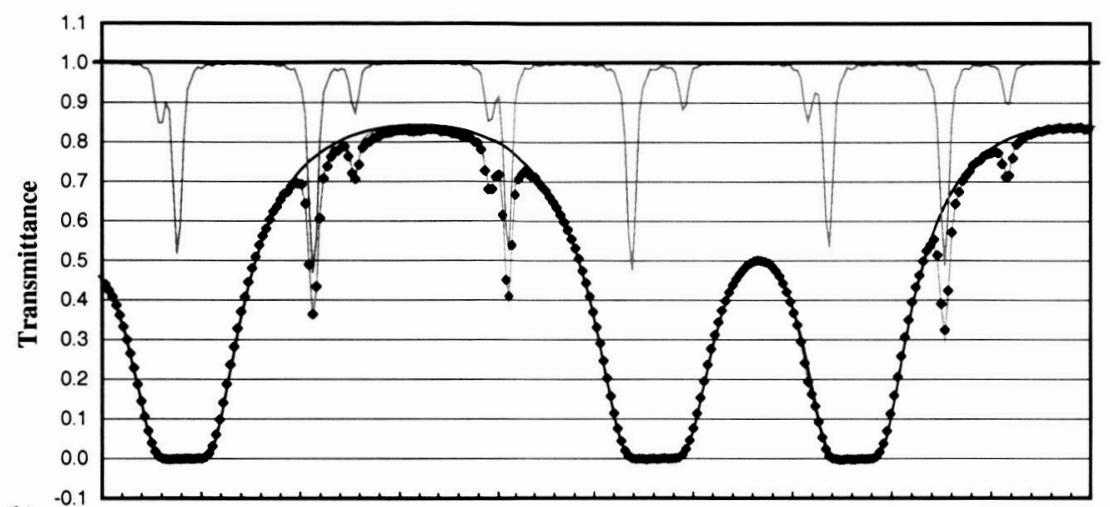

(b)

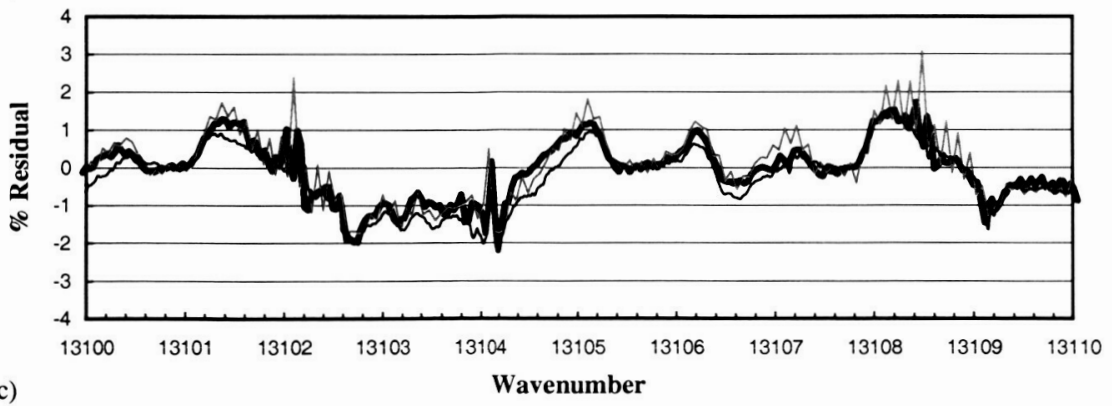

Fig. 3. (a) Photon path scale factors from the TMF spectra retrieved using Voigt lineshape plotted versus the airmasses at which they were taken. Negative and positive airmasses mean the spectra were taken in the morning and afternoon, respectively. Each day's results are marked differently. (b) An expanded view of the $13100-13110 \mathrm{~cm}^{-1}$ region for the same TMF spectrum shown in Fig. 1. (c) The spectral fitting residual of (b) (black line) together with residuals from fitting a Kitt Peak spectrum (pink line) and a Caltech spectrum (green dash line), demonstrating that the error pattern is not of instrumental origin. 


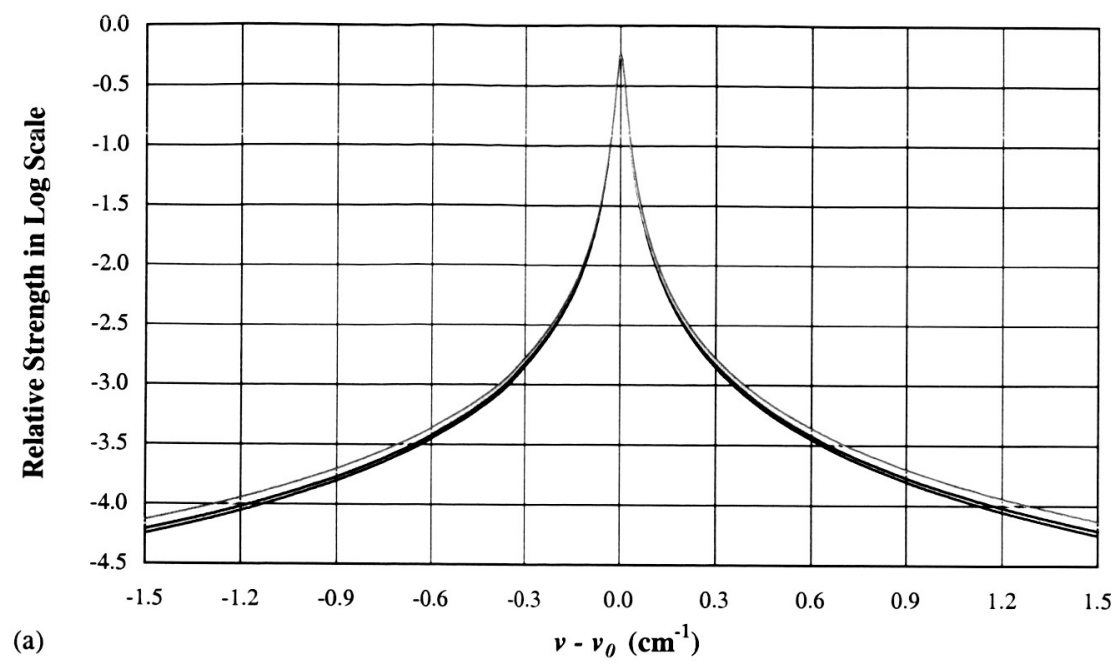

(a)

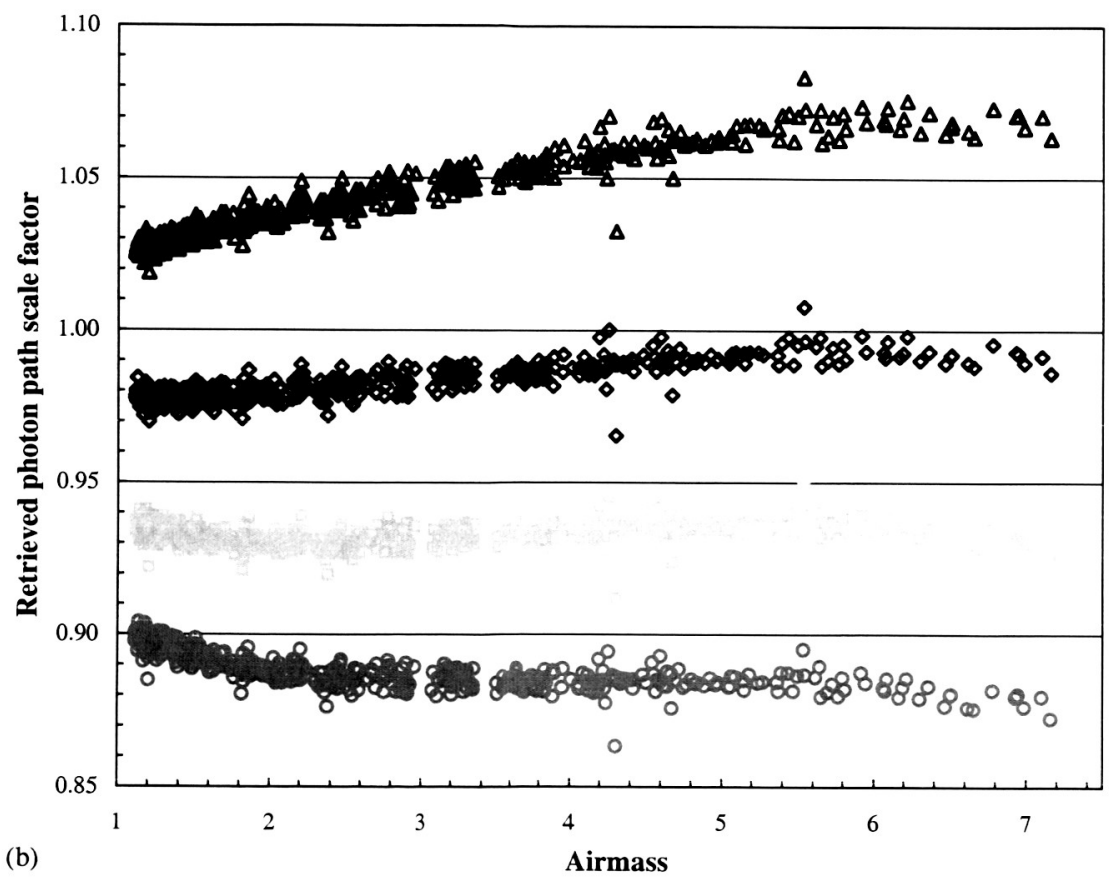

Fig. 4. (a) The comparison of Lorentz lineshape function (approximating Voigt function in the wings, in black) and super-Lorentz function with $\eta=1.977$ (red), 1.958 (yellow), and 1.940 (light blue), assuming a $0.015 \mathrm{~cm}^{-1} \mathrm{Doppler}^{-}$ width. The strength axis is in log scale. The far wing absorption clearly increases with decreasing $\eta$. The differences in the center are small and immaterial because the strong lines are saturated there. (b) Retrieved photon path scale factors using different lineshape functions versus airmass, using the same color scheme as in (a). 


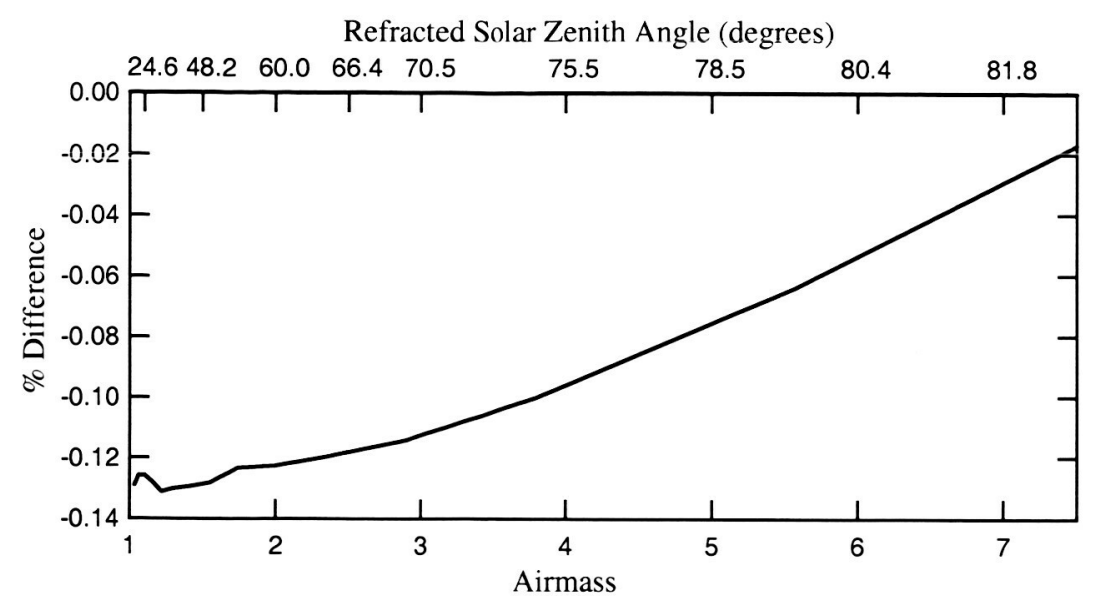

Fig. 5. Denote the integrated airmass from Kristensen [16] as $A M_{\mathrm{Kri}}$ and our 70-level discretized atmosphere calculation as $A M_{\mathrm{dis}}$. The relative difference between the two is defined as $\%$ Difference $=100 \times \frac{A M_{\mathrm{dis}}-A M_{\mathrm{Kri}}}{A M_{\mathrm{Kri}}}$ and plotted versus both airmass and refracted solar zenith angle.

values (0.97-1.00, should be unity). Nevertheless, without accurate laboratory information on the far-wing lineshape, it is impossible to ascertain if the retrieved non-unity scale factors are the result of spectroscopic, instrumental, or other errors.

The observed airmass dependence cannot be explained by errors introduced by the use of discrete levels in the ray path calculation. We compared our 70-level-discretized calculations of airmass with results of a numerical integration [17], both using the International Civil Aviation Organization (ICAO) standard atmosphere. The discrete calculation gives smaller photon paths, and the absolute difference increases almost linearly with airmass. However, the differences (shown in Fig. 5, 0.13\% at one airmass and $\sim 0.02 \%$ at seven airmasses) are small compared with the variation in $\mathrm{O}_{2}$ scale factors shown in Fig. 4(b). Moreover, correcting the ray-tracing error will worsen the spurious correlation between airmass and retrieval photon path, if we use Voigt lineshape or super-Lorentz lineshape of $\eta$ value larger than 1.977 .

Evaluation of achievable precision from current spectral retrieval technique is based on the scatter of the "flat region" in Fig. 4(b), analyzed using super-Lorentz function of $\eta=1.977$. For the 230 TMF spectra taken at airmasses less than 1.8 (Fig. 6), the mean of the retrieved scale factors is 0.9777 and the $1 \sigma$ is 0.0022 , sufficient to fulfill the requirement of calibrating $0.3 \%$ change in atmospheric $\mathrm{CO}_{2}$ over this relatively small variation in surface pressure $(\sim 10 \mathrm{mb})$. Observations made over a broader range of surface pressures would be helpful to further test the retrieval and could provide further insight about the physics of absorption in the A-band. In addition, in these mountain top observations, the water vapor column is small. This clearly will not be the case for soundings made at higher pressure. Because the influence of $\mathrm{H}_{2} \mathrm{O}$ on the lineshape is unknown, further retrieval error can be expected.

Although the super-Lorentz lineshape is imperfect for describing A-band lines, its better representation of the far wings may indicate the existence of collision-induced absorption (CIA), as has been previously speculated [18]. According to the statistical theory, super-Lorentz function of $\eta=2$ 


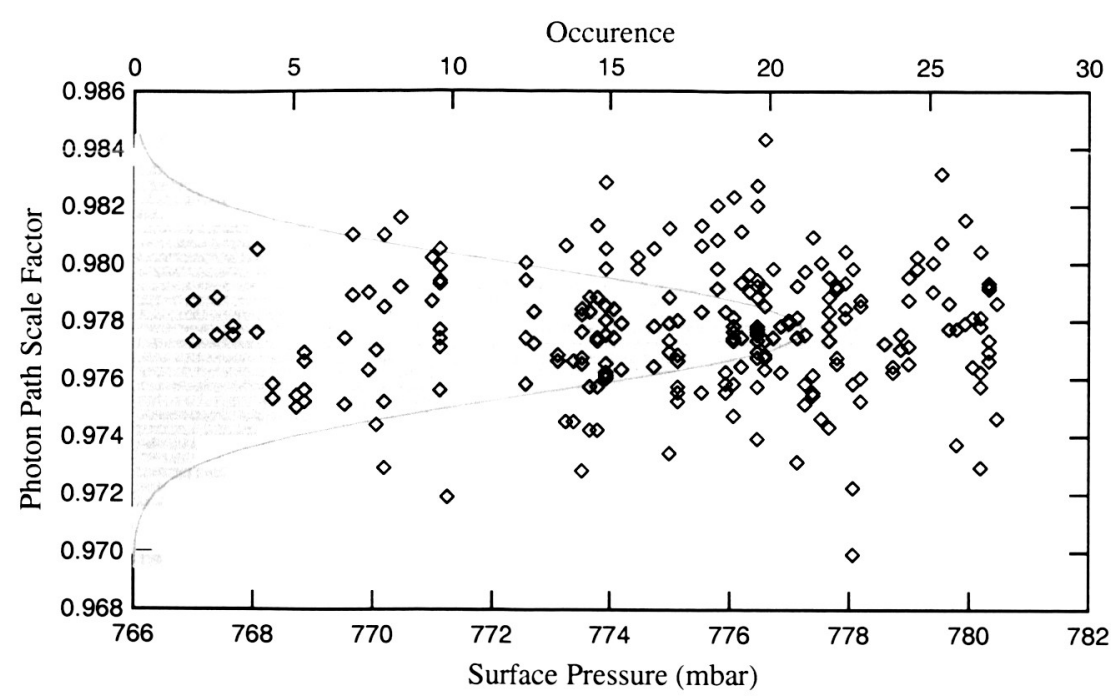

Fig. 6. Retrieved photon path scale factors from the low airmass $(<1.8)$ spectra using $\eta=1.977$ super-Lorentz function versus surface pressures at which they were taken. Corresponding histogram and the fitted Gaussian curve are in the background.

corresponds to dipole-dipole (e.g. $\mathrm{O}_{2}-\mathrm{O}_{2}$ or $\mathrm{O}_{2}-\mathrm{N}_{2}$ ) interaction and that of $\eta=1.75$ corresponds to dipole-quadrupole (e.g. $\mathrm{O}_{2}-\mathrm{O}_{4}$ ) interaction [16], so that the super-Lorentz lineshape of in-between $\eta$ values represents the mix of common absorption and CIA. Then again, the very small intensity of CIA in this region is immeasurable without an extremely long path cell in the lab. To derive the CIA from atmospheric spectra, by finding a lineshape function (could be similar to the multi-variable CKD model [19]) that minimizes the airmass dependence of the retrievals instead of absolute rms error of an individual fit, might be possible but difficult.

\section{Conclusion}

Using ground-based measurements of direct sunlight as a simple test, we have shown that photon paths can be retrieved with high precision $(\sim 0.2 \%)$ from high resolution FTS solar absorption spectra of the $\mathrm{O}_{2}$ A-band, over a significant SZA-driven (but not surface pressure) variation in airmass. This is achieved by virtue of: (1) the atmospheric $\mathrm{O}_{2}$ being extremely well mixed with an accurately known mole fraction; (2) the A-band lines being strong, numerous, and almost free of absorption by other gases; (3) the stable and self-calibrating nature of the FTS instrument; (4) the high signal-to-noise ratio of direct solar absorption spectra. Successful retrieval of photon paths will eliminate a major systematic error source of future remote sensing experiments using solar radiation as a light source, and greatly improve their precision in measuring many tropospheric species.

The major obstacle for further progress in this effort is that we cannot correctly represent the lineshape of the absorption in the A-band. Error in this parameterization results in spurious correlation between retrieved photon path scale factors and the airmasses at which the spectra were taken. 
Neither Voigt nor super-Lorentz lineshape is a completely correct description for the strong absorption lines in the A-band. Although we speculate that the near wing overestimation and the far wing underestimation could be due to line-mixing and continuum absorption, respectively, it is difficult to unambiguously determine the reasons for the poor spectral fits of open-path atmospheric spectra because there are too many unknowns (interfering absorption features, temperature/pressure varying along the line of sight, etc.). New laboratory measurements of the $\mathrm{O}_{2}$ A-band lineshape with a closed-path of at least $10 \mathrm{~km}$ are clearly needed.

\section{Acknowledgements}

We thank Linda Brown, Camy-Peyret and David Crisp for many helpful discussions, Rebecca Washenfelder for providing comparison spectra. Part of this work was carried out at the Jet Propulsion Laboratory, California Institute of Technology, under contract with NASA. Paul Wennberg, Geoff Toon, and Zhonghua Yang acknowledge support from NASA's ACMAP and Terrestrial Ecology Programs (NAG5-11739; NAG12247). Stan Sander, Rich Cageao, and Tom Pongetti acknowledge support from the NASA's Upper Atmosphere Research Program.

\section{References}

[1] Yamamoto G, Wark DQ. Discussion of letter by Hanel, RA-determination of cloud altitude from a satellite. J Geophys Res 1961;66:3596.

[2] O'Brien DM, Mitchell RM. Error-estimates for retrieval of cloud-top pressure using absorption in the A-band of oxygen. J Appl Meteorol 1992;31:1179-92.

[3] Kuze A, Chance KV. Analysis of cloud-top height and cloud coverage from satellites using the $\mathrm{O}_{2}$ A-band and B-bands. J Geophys Res Atmos 1994;99:14481-91.

[4] Asano S, Shiobara M, Uchiyama A. Estimation of cloud physical parameters from airborne solar spectral reflectance measurements for stratocumulus clouds. J Atmos Sci 1995;52:3556-76.

[5] Badaev VV, Malkevich MS. On the possibility of determining the vertical profiles of aerosol attenuation using satillite measurements of reflected radiation in the 0.76 um oxygen band. Izv Atmos Oceanic Phys 1978;14:722-7.

[6] Min Q, Harrison LC, Clothiaux EE. Joint statistics of photon path length and cloud optical depth: case studies. J Geophys Res Atmos 2001;106:7375-85.

[7] O'Brien DM, Mitchell RM, English SA, Da Costa GA. Airborne measurements of air mass from $\mathrm{O}_{2}$ A-band absorption spectra. J Atmos Ocean Technol 1998;15:1272-86.

[8] Nakajima H, Suzuki M, Yokota T, Sugita T, Itou Y, Kaji M, Araki N, Waragai K, Yamashita H, Kanzawa H, Sasano Y. Tangent height registration for the solar occultation satellite sensor ILAS: a new technique for Version 5.20 products. J Geophys Res Atmos 2002; 107: Article no 8215.

[9] Kuang ZM, Margolis J, Toon G, Crisp D, Yung Y. Spaceborne measurements of atmospheric $\mathrm{CO}_{2}$ by high-resolution NIR spectrometry of reflected sunlight: an introductory study. Geophys Res Lett 2002; 29: Article no 1716.

[10] Cageao RP, Blavier JF, McGuire JP, Jiang YB, Nemtchinov V, Mills FP, Sander SP. High-resolution Fourier-transform ultraviolet-visible spectrometer for the measurement of atmospheric trace species: application to OH. Appl Opt 2001;40:2024-30.

[11] Gamache RR, Goldman A, Rothman LS. Improved spectral parameters for the three most abundant isotopomers of the oxygen molecule. JQSRT 1998;59:495-509.

[12] Ritter KJ, Wilkerson TD. High-resolution spectroscopy of the oxygen A-band. J Mol Spectrosc 1987;121:1-19.

[13] Yang SF, Canagaratna MR, Witonsky SK, Coy SL, Steinfeld JI, Field RW, Kachanov AA. Intensity measurements and collision-broadening coefficients for the oxygen $\mathrm{A}$ band measured by intracavity laser absorption spectroscopy. J Mol Spectrosc 2000;201:188-97. 
[14] Brown LR, Plymate C. Experimental line parameters of the oxygen A-band at $760 \mathrm{~nm}$. J Mol Spectrosc 2000;199: 166-79.

[15] Newman SM, Orr-Ewing AJ, Newnham DA, Ballard J. Temperature and pressure dependence of line widths and integrated absorption intensities for the $\mathrm{O}_{2} \mathrm{a}^{1} \Delta_{\mathrm{g}}-\mathrm{X}^{3} \Sigma_{\mathrm{g}}^{-}(0,0)$ transition. J Phys Chem A 2000;104:9467-80.

[16] Hirono M, Nakazawa T. The shape of spectral lines with combined impact and statistical broadenings. J Phys Soc Jpn 1982;51:265-8.

[17] Kristensen LK. Astronomical refraction and airmass. Astron Nachr 1998;319:193-8.

[18] Mlawer EJ, Clough SA, Brown PD, Stephen TM, Landry JC, Goldman A, Murcray FJ. Observed atmospheric collision-induced absorption in near-infrared oxygen bands. J Geophys Res Atmos 1998;103:3859-63.

[19] Clough SA, Kneizys FX, Davies RW. Line shape and the water vapour continuum. Atmos Res 1989;23:229-41. 